\title{
Inhibition of Pre-B Cell Colony Enhancing Factor Reduces Lung Injury in Rats Receiving Cardiopulmonary Bypass
}

This article was published in the following Dove Press journal: Drug Design, Development and Therapy

\section{Chao Lu \\ Wei Yang \\ Jianliang Zhou \\ Zulei Zhang $\mathbb{D}$ \\ Yi Gong \\ Fajia $\mathrm{Hu}$ \\ Wenpeng Yu \\ Xiao Dong}

Department of Cardiovascular Surgery, The Second Affiliated Hospital of Nanchang University, Nanchang, Jiangxi 330006, People's Republic of China
Correspondence: Xiao Dong Email dongxiaol45@163.com
Objective: Pre-B cell colony enhancing factor (PBEF) is an important proinflammatory cytokine involved in acute lung injury. However, whether PBEF participates in lung injury caused by cardiopulmonary bypass (CPB) is still unknown. This study aimed to investigate the effects of silencing PBEF on lung injury and the sodium and water transport system in rats receiving $\mathrm{CPB}$.

Methods: Morphological changes in lung tissues were evaluated using hematoxylin and eosin (H\&E) staining. PBEF was detected using immunohistochemistry. The sodium and water transport system-related proteins and cellular signaling pathways were detected by Western blotting.

Results: Rats receiving CPB (model group) had more severe alveolar wall damage and higher expression of PBEF in free form than the control rats. Western blotting showed that the expression of PBEF, surfactant protein D (SP), aquaporin (AQP) 1, AQP5, and epithelial sodium channel $(\mathrm{ENaC})$ was significantly higher in the lung tissue of $\mathrm{CPB}$ rats than control rats. By contrast, adenovirus-encoding sh-PBEF significantly reduced the expression of PBEF, SP, AQP1, AQP5, and ENaC in the lung tissues of rats treated with CPB. The phosphorylation levels of extracellular signal-regulated protein kinases 1 and 2 (ERK1/2), protein kinase B (AKT), and p38 mitogen-activated protein kinase (MAPK) were significantly increased in the lung tissue of rats that received $\mathrm{CPB}$, and were downregulated by adenovirus-encoding sh-PBEF.

Conclusion: Adenovirus-encoding sh-PBEF could reduce lung injury and repair the sodium-water transport system in rats receiving $\mathrm{CPB}$, likely through reducing MAPK, ERK1/2, and Akt signaling pathways.

Keywords: pre-B cell colony enhancing factor, epithelial sodium channel, aquaporin, cardiopulmonary bypass

\section{Introduction}

Cardiopulmonary bypass (CPB) is a common method used to minimize bleeding during surgery ${ }^{1}$ and could reduce neurological morbidity rates. ${ }^{2}$ However, this procedure carries a high risk of brain injury and is frequently associated with postoperative lesions. ${ }^{3,4}$ Pulmonary edema is the central link for acute lung injury (ALI) after $\mathrm{CPB},{ }^{5}$ primarily because of the increase of alveolar-capillary permeability and decrease of alveolar fluid clearance. The failure of timely clearance of alveolar fluid is the pathological basis for serious complications such as decreased postoperative pulmonary function and lung perfusion. ${ }^{6,7}$ 
The sodium-water transport system of alveolar epithelial cells is mainly composed of the epithelial sodium channel $(\mathrm{ENaC})$ and aquaporins (AQPs). $\mathrm{ENaC}$ in the alveolus cavity absorbs $\mathrm{Na}^{+}$, and then pumps $\mathrm{Na}^{+}$into the lung interstitium through the surface of the basal side. ${ }^{8}$ Because $\mathrm{Na}^{+}$is pumped out of the cells, a significant electrochemical gradient is created in the cells, accompanied by the passive absorption of water and chloride ions $\left(\mathrm{Cl}^{-}\right) .{ }^{9,10}$ In addition to the active transport of sodium, water is also excreted through AQPs present on the surface of alveolar epithelial cells. ${ }^{11}$

A recent study reported on the signaling pathways that regulate the expression of $\mathrm{ENaC}$ and $\mathrm{AQPs} .{ }^{8} \mathrm{ENaC}$ and TGF$\beta 1$ can reduce the expression of the $\alpha$-subunit of $\mathrm{ENaC}$ in human and rat alveolar epithelial cells through the Erkl/2 signaling pathway, and inhibit amiloride-sensitive sodium transport. ${ }^{12}$ In a study of lipopolysaccharides (LPS)-induced acute lung injury (ALI), 17 $\beta$-estradiol reduced pulmonary edema and upregulated the expression of $\mathrm{ENaC}$ through the phosphoinositide 3-kinase (PI3K)/protein kinase B (AKT)/ glucocorticoid-inducible kinase 1 (SGK1) signaling pathway in mice. ${ }^{13}$ Interleukin (IL)-6, IL-8, tumor necrosis factor (TNF)- $\alpha$, and other inflammatory cytokines also inhibited the expression of AQP1 through MAPKs signaling pathway, resulting in imbalance and dysfunction of fluid transport. ${ }^{14}$

The pre- $\mathrm{B}$ cell colony enhancing factor (PBEF) is a nicotinamide phosphoribosyl transferase. ${ }^{15}$ PBEF typically acts as an $\mathrm{NAD}^{+}$synthesis rate-limiting enzyme and an insulin-like factor and as a regulatory factor of inflammation and immune process. ${ }^{16-18}$ It has been reported that the expression of PBEF increased with ALI, and the expression of TNF-a, IL-1 $\beta$, transforming growth factor beta 1 (TGF- $\beta 1$ ), and other inflammatory factors in the lung were upregulated, thereby prohibiting sodium-water transport system of alveolar epithelial cells, evidenced by the decreased expression of $\mathrm{ENaC}$ and AQPs. ${ }^{19-21}$

In this study, the pathological changes in lung tissue were observed in rats receiving $\mathrm{CPB}$, and the regulatory effect of PBEF on the sodium-water transport system in lung tissue were investigated, in addition to the potential signaling pathways. This study will likely have important implications for lung injury remedies after CPB.

\section{Materials and Methods}

\section{Animals and Treatments}

Male Sprague-Dawley rats (weight: 300-400 g) were purchased from Hunan Slake Jingda Experimental Animal
Co., Ltd. (license No. SCXK [Hunan] 2016-0002). All animal experiments were performed in accordance with the Guide for the Care and Use of Laboratory Animals published by the Ministry of Science and Technology of China (2006), and these experiments were approved by the Ethics Committee of the Second Affiliated Hospital of Nanchang University.

The rats were randomly divided into six groups after one week of adaptive feeding: a control group (rats were anesthetized and intubated without circulation); a model group (30-min CPB); an LPS group; an Adenovirusencoding sh-PBEF+LPS group; an adenovirus vector + model group (adenovirus was injected one week before $\mathrm{CPB}$ ); and an Adenovirus-encoding sh-PBEF+model group (adenovirus was injected one week before deep hypothermic circulatory arrest $[\mathrm{DHCA}]$ and $\mathrm{CPB}$ ). The dosage of injected adenovirus was $2 \times 10^{8} \mathrm{PFU} /$ rat. Adenovirus was obtained from Wuhan GeneCreate Bioengineering Co., Ltd. (Wuhan, China). The sequence of sh-PBEF was 5'-GTACAGGTTACTCACTA TAAATTCAAGATTAGTAGAPTTGTATTTTT-3', which was synthesized by Sangon Biotech (Shanghai, China).

\section{CPB Procedure}

The rats were anesthetized by intraperitoneal injection of $3 \%$ pentobarbital $(50 \mathrm{mg} / \mathrm{kg})$. The right external jugular vein and right internal carotid artery were exposed through a longitudinal incision of the neck, and the right femoral artery was exposed through an incision in the right groin. The right femoral artery was catheterized and connected with a pressure sensor to measure blood pressure. The right carotid artery was catheterized and perfused with an indwelling venous catheter needle (20G). The right external jugular vein central venous catheter was used to establish a vein channel. Heparin $(500 \mathrm{IU} / \mathrm{kg})$ was injected into the right external jugular vein for systemic heparinization. Then, the rats were connected with the CPB apparatus, pre-charged with the solution: $10 \mathrm{~mL}$ substitute plasma (Hess), $0.5 \mathrm{~mL} \mathrm{5 \%} \mathrm{NaHCO}_{3}$, and $0.5 \mathrm{~mL} 20 \%$ mannitol. The deep hypothermia circulatory arrest procedure was induced in rats. Briefly, after cooling down the body temperature to $20-25^{\circ} \mathrm{C}$ for $30 \mathrm{~min}$ by using a heat exchanger and topical cooling with ice bags and a cooling blanket, DHCA confirmed by asystole were maintained for $30 \mathrm{~min}$. After DHCA, CPB was reinstituted, and rats were rewarmed to rectal temperatures of at least $35.5^{\circ} \mathrm{C}$ over $40 \mathrm{~min}$ as previously described. ${ }^{22}$ Electrocardiogram (ECG) and anal temperature were routinely monitored 
during the operation. When stable respiration, hemodynamics, and an anal temperature of $36^{\circ} \mathrm{C}$ were achieved, the machine was stopped and protamine and heparin were injected intravenously. The rats were allowed to feed freely in an oxygen-sufficient environment for $2 \mathrm{~h}$, and heart rate and mean arterial pressure were monitored during the surgery. Two hours after operation, the rats were decapitated and the lung tissues were collected for further analysis. After transfection, SD rats were injected with $4 \mathrm{mg} / \mathrm{kg}$ LPS through the tail vein. Follow-up experiments were carried out $4 \mathrm{~h}$ after the injection.

\section{Determination of the Weight Ratio of Dry Tissue to Wet Tissue}

After weighing the lung tissue, it was placed in the incubator at $60^{\circ} \mathrm{C}$ for $24 \mathrm{~h}$ for baking. Then, the weight of the dried lung tissue was also recorded. The dry:wet (D/W) ratio of the lung weight was calculated. The average value was measured three times, and the ratio was calculated with the wet weight.

\section{Detection of $\mathrm{Na}^{+}-\mathrm{K}^{+}$-ATPase Activity}

$\mathrm{Na}^{+}-\mathrm{K}^{+}$-ATPase activity was measured according to the manufacturer's instructions of the $\mathrm{Na}^{+}-\mathrm{K}^{+}$-ATPase activity kit (Solarbio, Beijing, China).

\section{HE Staining}

The lung tissue was fixed in $4 \%$ PFA overnight at $4{ }^{\circ} \mathrm{C}$. The tissues were dehydrated in 70\%, 80\%, and 90\% ethanol solution; a mixture of pure ethanol and xylene for 15 min; xylene I for $15 \mathrm{~min}$; and xylene II for $15 \mathrm{~min}$. The tissues were then placed in the mixture of xylene and paraffin for $15 \mathrm{~min}$, followed by paraffin I and paraffin II for 50 and $60 \mathrm{~min}$, respectively. The tissues were embedded in paraffin, sectioned into $20-\mu \mathrm{m}$-thick sections, and baked and then dewaxed and hydrated. The sections were then stained in an aqueous solution of hematoxylin for $3 \mathrm{~min}$ and eosin for $3 \mathrm{~min}$. Last, the slides were sealed and imaged under a light microscope. At least four fields were taken from each slide. Lung injury scores were quantified as previously described. ${ }^{23}$

\section{Immunohistochemistry}

The sections were prepared as stated above and stained using monoclonal antibodies against PBEF (1:200, 117761-AP, Proteintech) at $4^{\circ} \mathrm{C}$ overnight. Slides were incubated with horseradish peroxidase (HRP)-labeled goat anti- rabbit $\operatorname{IgG}$ secondary antibody $(1: 10,000$; catalog no A16104SAMPLE; Thermo Fisher Scientific, Inc., Waltham, MA, USA) and Alexa Fluor 593 goat antimouse IgG (Life Technologies, Carlsbad, CA, USA) for $30 \mathrm{~min}$ at room temperature. The sections were observed and photographed using a light microscope (BX51, Olympus, Japan). At least four fields were taken from each slide. The quantification data of protein expression were calculated based on the literature. ${ }^{24}$

\section{Western Blotting}

The protein was extracted from liver tissues, and protein concentration was determined using the bicinchoninic acid assay. Briefly, $20 \mu \mathrm{g}$ protein in each group was processed in $12 \%$ sodium dodecyl sulfate-polyacrylamide gel electrophoresis. After transferring onto polyvinylidene fluoride (PVDF) membrane, non-specific staining was blocked by $5 \%$ skim milk. The membrane was incubated with the following antibodies overnight at $4^{\circ} \mathrm{C}$ : rabbit anti-PBEF (1:2000, 11776-1-AP, Proteintech); rabbit anti-ERK1/2 (1:1000, 16443-1-AP, Proteintech); rabbit anti-p-ERK1/2 (1:500, bs-3016R, Bioss); rabbit anti-p38MAPK (1:1000, bs-0637R, Bioss); rabbit anti-p-p38MAPK (1:1000, bs5477R, Bioss); rabbit anti-AKT (1:500, ab8805, Abcam); rabbit anti-p-AKT (1:1000, bs-2720R, Bioss); rabbit antiAQP1 (1:1000, bs-1506R, Bioss); rabbit anti-AQP5 (1:1000, AF5169, Affinity); rabbit anti-ENaC (1:1000, bs2957R, Bioss); rabbit anti-surfactant protein D (SP) (1:1000, ab220422, Abcam); and mouse monoclonal antiGAPDH (1:2000, TA-08, ZSBio).

\section{Statistical Analysis}

Data were expressed at mean and standard deviation and analyzed by SPSS 19.0. Considering CPB-related lung injury and LPS-related one are independent considering the possibly different biological behaviors in lung injury, we did independent analysis regarding CPB-related groups and LPS-related groups. The data were analyzed by oneway ANOVA followed by Newman-Keuls method as the post-hoc test. $\mathrm{P}<0.05$ was considered to indicate statistical significance.

\section{Results}

\section{Heart Rate and Mean Arterial Pressure}

After initiation of $\mathrm{CPB}$, the vital signs of rats were stable. Thirty minutes after $\mathrm{CPB}$, the body temperature of rats was physically cooled $\left(20-23^{\circ} \mathrm{C}\right)$ to deepen hypothermia; 
Table I Heart Rate and Mean Arterial Pressure During Cardiopulmonary Bypass in Rats (Mean \pm SD)

\begin{tabular}{|l|l|l|}
\hline & Heart Rate (Beats/min) & Mean Arterial Pressure (mmHg) \\
\hline Cardiopulmonary bypass & $338.75 \pm 65.721$ & $101.23 \pm 16.375$ \\
After cooling down & $91.724 \pm 23.672$ & $82.23 \pm 9.461$ \\
After rewarming & $319.764 \pm 51.658$ & $94.75 \pm 13.382$ \\
\hline
\end{tabular}

then, deep hypothermia circulatory arrest operation was begun. The operation center rate was greatly simplified, and the blood pressure and heart rate of the rats returned to the previous level after the temperature was restored. After CPB was terminated and the catheter withdrawn, the rats were normal. After the anesthesia wore off, the animals had ad libitum access to food and water. The heart rate and mean arterial pressure are shown in Table 1.

Compared with controls, the ratio of dry:wet weight increased in the CPB group and LPS group. Sh-PBEF reduced the increase of dry:wet ratio caused by $\mathrm{CPB}$, but not LPS (Figure 1).

\section{Pathological Changes of Lung Tissues}

The lung alveoli of rats receiving CPB and treated by LPS were apparently impaired compared to the control group (Figure 2). By contrast, no pathological changes were observed in the lung tissues of control, blank, and PBEF shRNA rats. Adenovirus-encoding sh-PBEF, but not the vector, obviously reduced the damage of lung tissue caused by CPB or LPS treatment.

\section{Adenovirus-Encoding sh-PBEF Reduced the Increase of PBEF Caused by CPB}

The immunohistochemical results of PBEF expression in lung tissue of rats are shown in Figure 3. PBEF was widely expressed in lung tissue; the expression was higher in the endobronchial wall and in some free cells in the lungs of rats receiving CPB or LPS treatment. By contrast, the increased expression of PBEF by CPB was downregulated by adenovirus-encoding sh-PBEF.

\section{Adenovirus-Encoding sh-PBEF Reduced the Increase of $\alpha$-Subunit of PBEF, SP, AQPI, AQP5, and ENaC in the Lung}

\section{Tissue}

The protein expression of $\alpha$-subunit of PBEF, SP, AQP1, $\mathrm{AQP} 5$, and $\mathrm{ENaC}$ in the lung are shown in Figure 4. Compared to the controls, rats that received CPB or LPS treatment showed significantly increased expression of
PBEF, SP, AQP1, AQP5, and ENaC in the lung tissue. By contrast, the increased expression of PBEF, SP, AQP1, and AQP5 caused by CPB or LPS was reduced by sh-PBEF. ShPBEF also reduced the increase of ENaC caused by LPS.

\section{Effects of Adenovirus-Encoding sh-PBEF on $\mathrm{Na}^{+}-\mathrm{K}^{+}$-ATPase Activity of Lung}

\section{Tissue}

$\mathrm{Na}^{+}-\mathrm{K}^{+}$-ATPase activity in lung tissue was up-regulated in CPB and LPS groups, while sh-PBEF down-regulated the activity caused by LPS, but not by CPB (Figure 5).

\section{Adenovirus-Encoding sh-PBEF Reduced the Increase of Phosphorylation of ERKI/}

\section{2, Akt, and MAPK (P38)}

The phosphorylation levels of ERK1/2, Akt, and p38MAPK in lung tissues of rats receiving CPB or LPS treatment were significantly increased compared with

\section{Wet-Dry weight ratio}

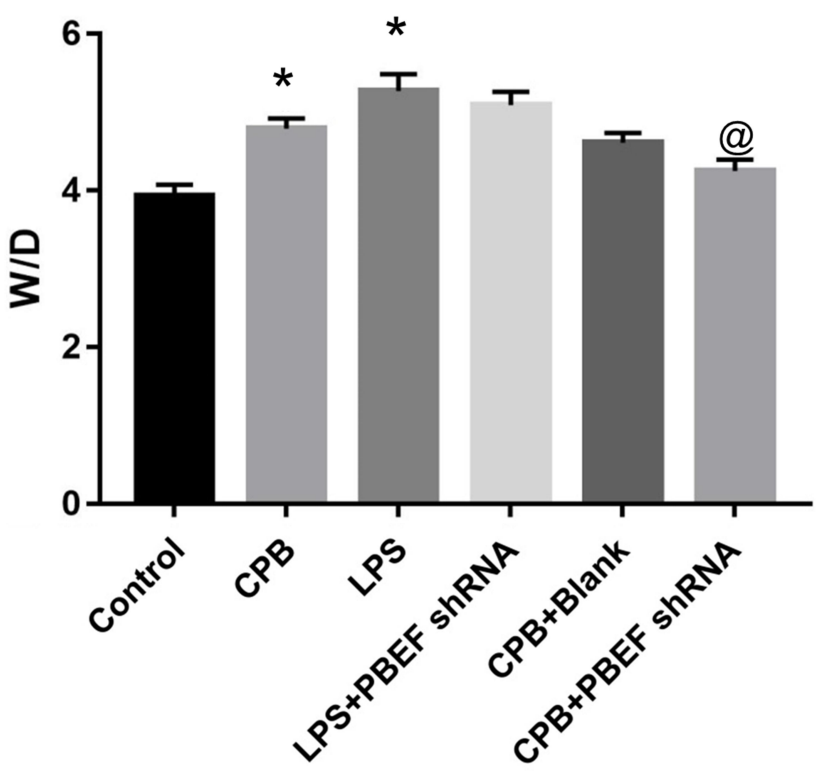

Figure I Adenovirus-encoding sh-PBEF reduced the dry:wet $(D / W)$ tissue ratio vs control, *P<0.05; vs CPB+Blank, ${ }^{\circledR} \mathrm{P}<0.05$.

Abbreviations: PBEF, pre-B cell colony enhancing factor; $C P B$, cardiopulmonary bypass. 
A

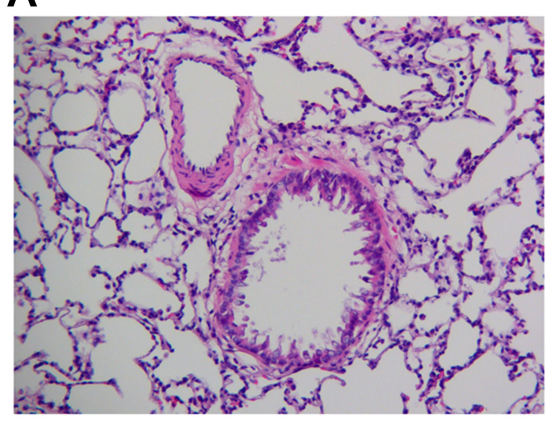

B

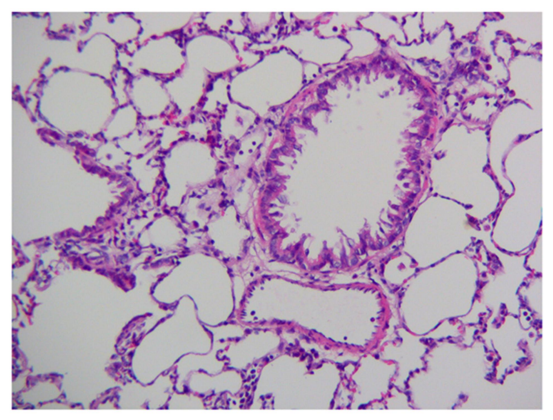

C

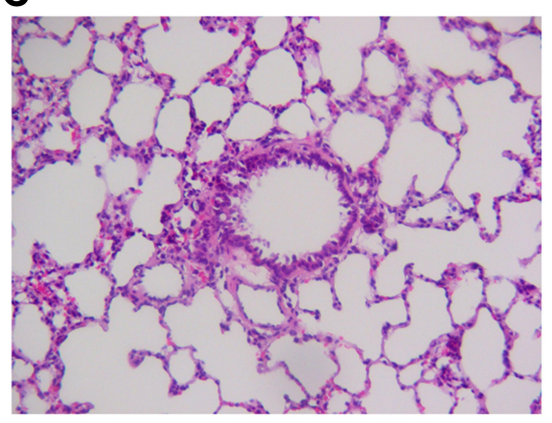

D

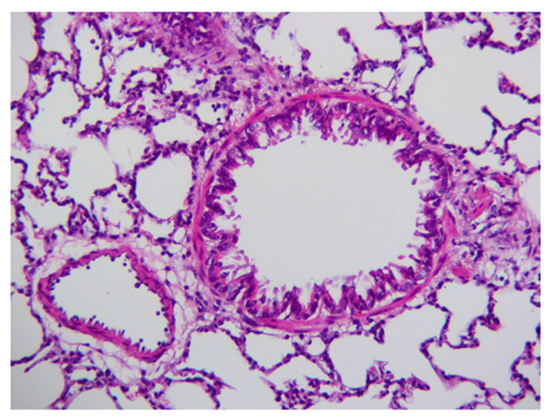

E

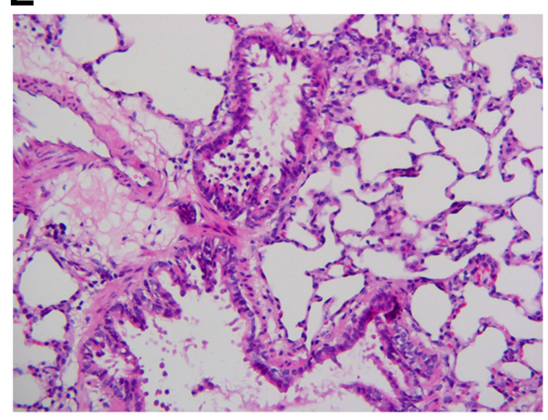

F

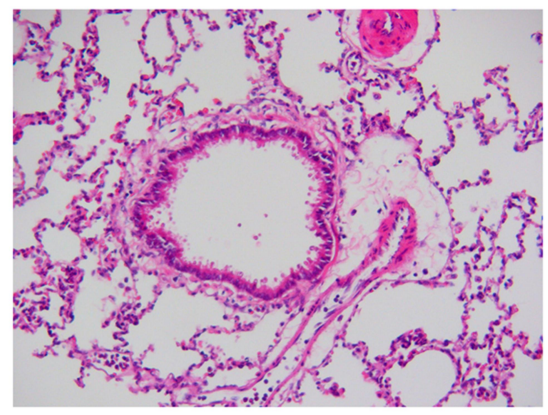

G

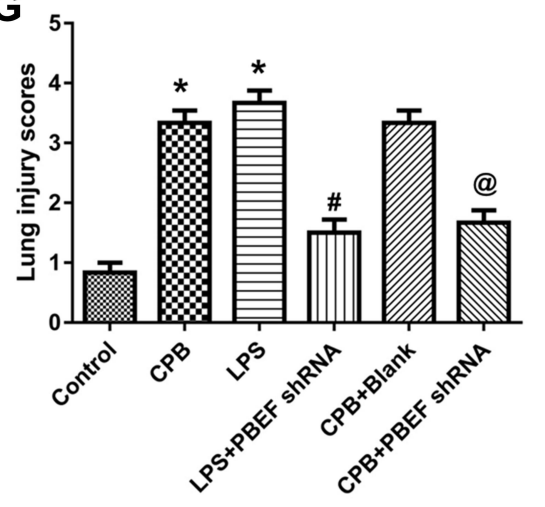

Figure 2 Pathological changes of lung tissue. HE staining was applied to detect the pathological changes of lung tissue. The lung alveoli of rats receiving CPB and LPS injection were damaged, while pathological changes of lung tissues in control, blank, and PBEF shRNA were not observable. Adenovirus-encoding sh-PBEF reduced the damage of lung tissue caused by CPB or LPS. (A): Control; (B): CPB; (C): LPS; (D): LPS+PBEF shRNA; (E): CPB+Blank; (F): CPB+PBEF shRNA (Magnification: x200); (G) Lung injury scores vs control, ${ }^{*} \mathrm{P}<0.05$; vs LPS, ${ }^{*} \mathrm{P}<0.05$; vs $\mathrm{CPB}+\mathrm{Blank}$, ${ }^{@} \mathrm{P}<0.05$.

Abbreviations: PBEF, pre-B cell colony enhancing factor; CPB, cardiopulmonary bypass.

control. However, the increased phosphorylation levels of ERK1/2, Akt, and p38MAPK were significantly reduced by Adenovirus-encoding sh-PBEF (Figure 6).

\section{Discussion}

We previously established a hypoxia model of human umbilical vein endothelial cells in vitro to confirm that PBEF would increase in expression during hypoxia. Cell permeability increases, and adenovirus-encoding sh-PBEF can reduce the changes in permeability ${ }^{25}$ through the MAPK and ERK signaling pathways. ${ }^{26}$ In addition, PBEF could inhibit sodium-water transport system- related protein expression in rat type I and type II alveolar epithelial cells. ${ }^{8,27}$

Nevertheless, the previous conclusions are mostly based on in vitro cell experiments. To verify the in vivo relationship between PBEF and the active removal of pulmonary edema fluid mediated by the sodium-water transport system, it is necessary to construct animal CPB models. In this present study, a rat CPB model was utilized to evaluate the function of PBEF. According to Waterbury et $\mathrm{al}^{28}$ the establishment of a rat DHCA model successfully simulated the circulatory arrest process in clinical CPB. We also adopted a physical cooling method to 
A

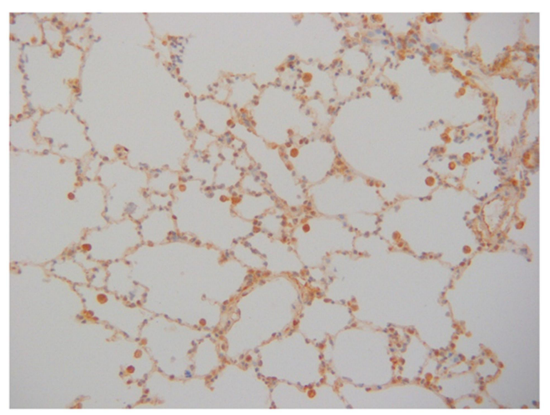

B

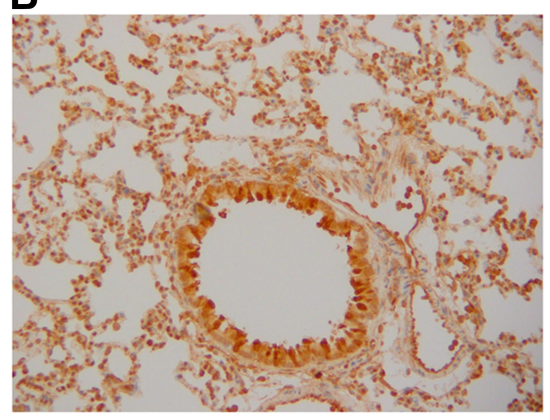

C

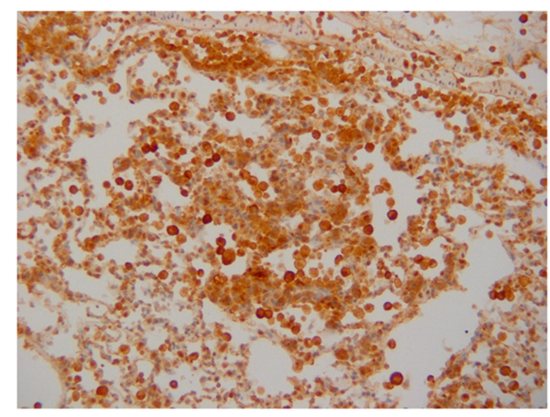

D

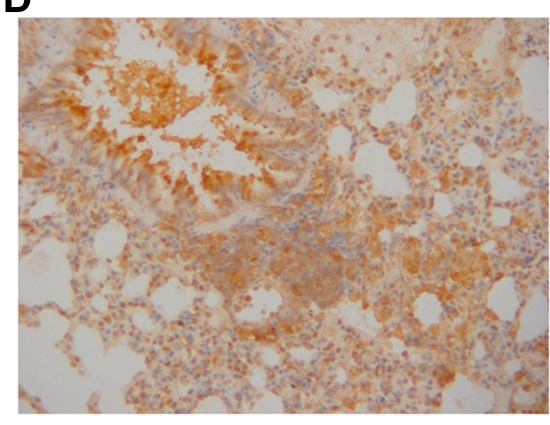

E

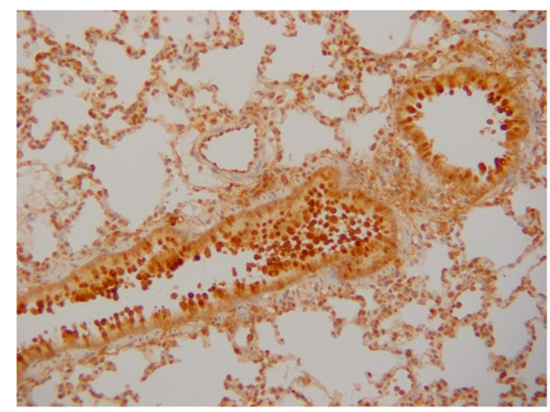

F

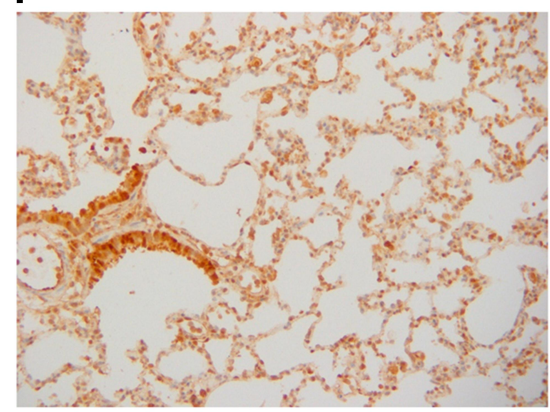

G

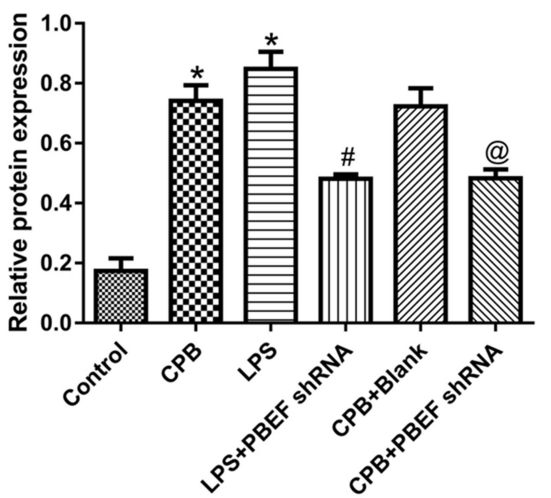

Figure 3 Adenovirus-encoding sh-PBEF reduced the increase of PBEF caused by CPB. PBEF was widely expressed in lung tissue in a free form. Compared with the rats without CPB, those with CPB had higher expression of PBEF. By contrast, the increase of PBEF by CPB was downregulated in the Adenovirus-encoding sh-PBEF-treated group (PBEF positive: brown; nucleus: blue). (A): Control; (B): CPB; (C): LPS; (D): LPS+PBEF shRNA; (E): CPB+Blank; (F): CPB+PBEF shRNA (Magnification: $\times 200)$; (G) Relative protein expression vs control, *P<0.05; vs LPS, ${ }^{*} \mathrm{P}<0.05$; vs $\mathrm{CPB}+\mathrm{Blank}$, ${ }^{\circledR} \mathrm{P}<0.05$.

Abbreviations: PBEF, pre-B cell colony enhancing factor; $\mathrm{CPB}$, cardiopulmonary bypass.

greatly simplify the rat heartbeat. It can make the pumping volume of the rat's heart beating very little, and the pulmonary artery perfusion is greatly reduced. This DHCAassisted CPB method simulates the lung injury in clinical $\mathrm{CPB}$ to the greatest extent. To similarly compare whether the CPB model constructed in the experiment can cause lung injury, we utilized a model of inflammatory lung injury by tail vein injection of LPS. Use of LPS is an established method of inflammatory reaction, ${ }^{29}$ especially inducing lung injury. ${ }^{30}$ The HE staining showed that LPS can cause severe swelling of the lung interstitium and structural deformation of the alveolar wall in rats. The pathological sections of rats that received CPB also show apparent changes; however, the degree was lighter than LPS, and biochemical analysis showed that PBEF was highly expressed in both models of lung injury. It can be seen that the CPB model constructed in this experiment most likely caused a certain degree of edema in the lung tissue of rats.

As an immune cytokine, PBEF is highly expressed in neutrophils and lymphoid cells. ${ }^{31}$ In this experiment, the immunohistochemical results of lung tissue that caused 


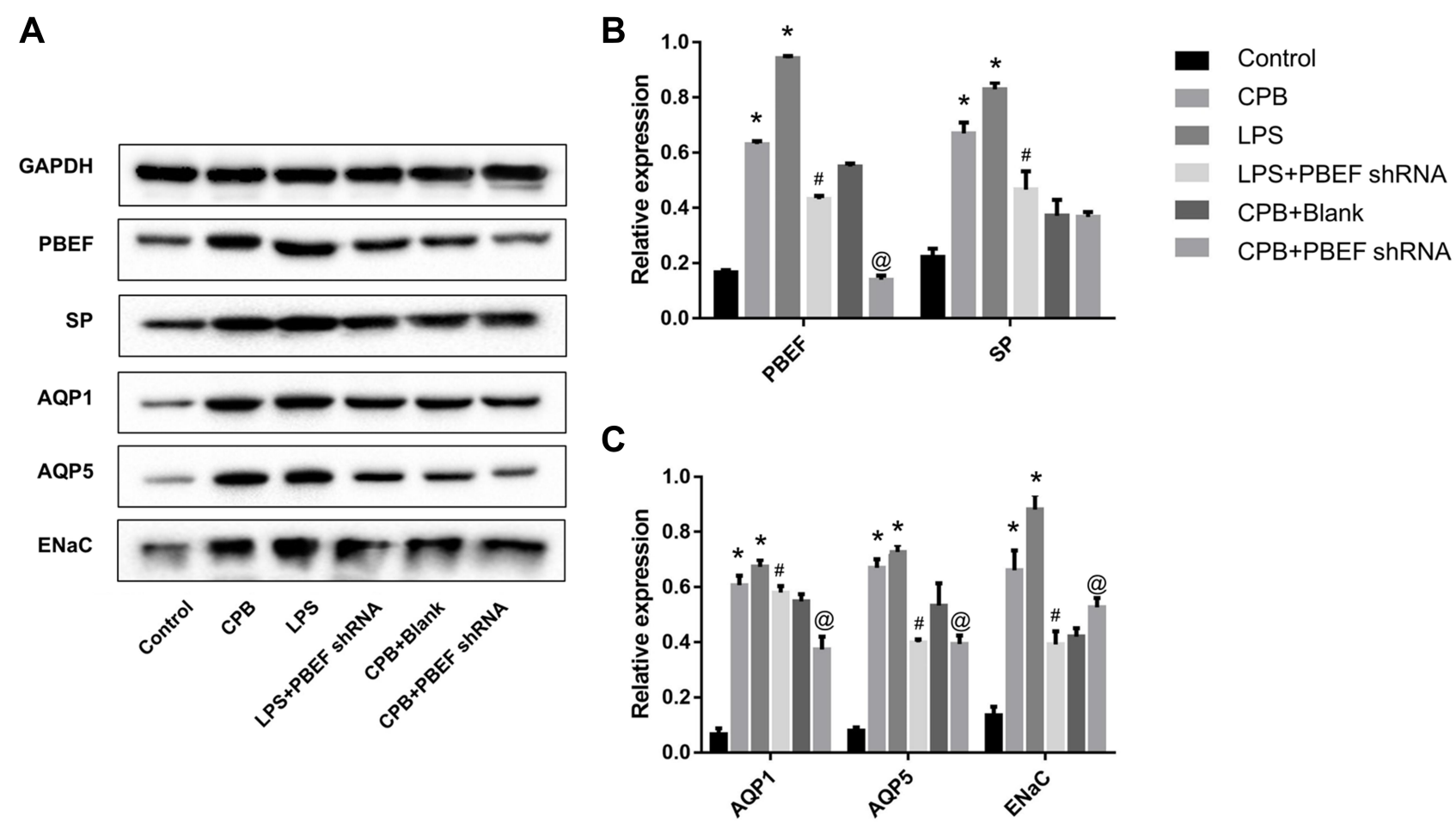

Figure 4 Adenovirus-encoding sh-PBEF reduced the increase of $\alpha$-subunit of PBEF, SP, AQPI, AQP5, and ENaC in lung tissue. (A) Representative blots; (B) Relative expression of PBEF and SP; (C) Relative expression of AQPI, AQP5, and ENaC; Original blots were shown in Supplemental figure I; vs control, *P<0.05; vs LPS, ${ }^{\#}$ < 0.05 ; vs $\mathrm{CPB}+$ Blank, ${ }_{\mathrm{P}}<0.05$.

Abbreviations: PBEF, pre-B cell colony enhancing factor; $\mathrm{CPB}$, cardiopulmonary bypass.

\section{$\mathrm{Na}+-\mathrm{K}+-\mathrm{ATP}$ Enzyme activity}

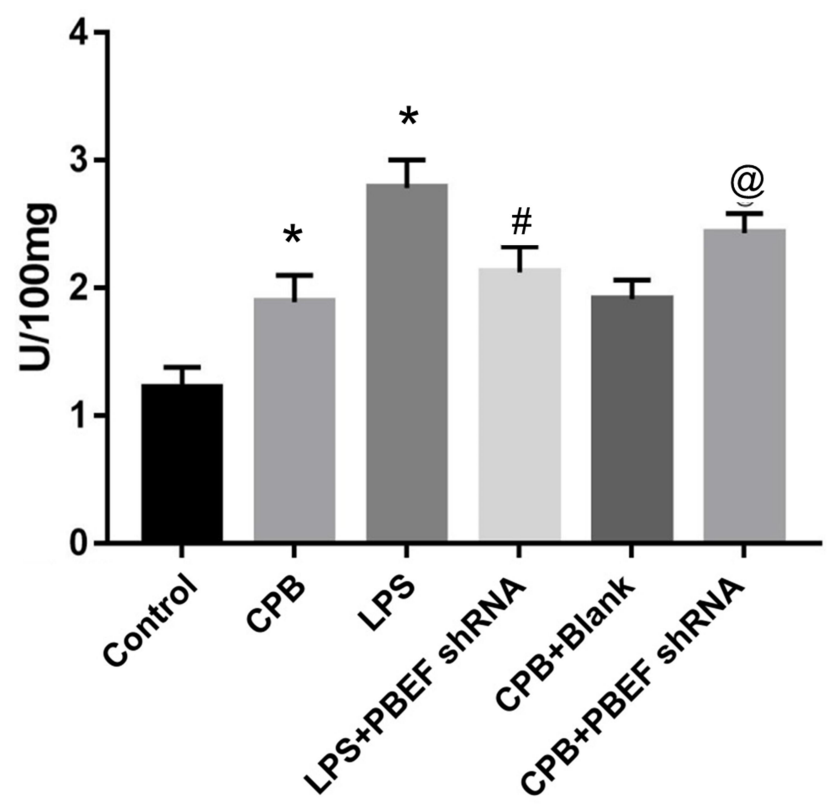

Figure 5 Effects of adenovirus-encoding sh-PBEF on $\mathrm{Na}^{+}-\mathrm{K}^{+}$-ATPase activity of lung tissue vs control, ${ }^{*} \mathrm{P}<0.05$; vs LPS, ${ }^{*} \mathrm{P}<0.05$; vs $\mathrm{CPB}+\mathrm{Blank}$, ${ }^{@} \mathrm{P}<0.05$.

Abbreviations: $\mathrm{PBEF}$, pre-B cell colony enhancing factor; $\mathrm{CPB}$, cardiopulmonary bypass. lung damage can be observed in the alveolar PBEF. There are varying degrees of expression in the wall, bronchial wall, or alveoli. It can be inferred that cells with high expression of PBEF in the lung tissue are neutrophils or lymphocytes transferred from the capillaries to the alveoli, which reflects a certain degree of inflammation in the lung tissue.

Reducing PBEF expression inhibited the phosphorylation of ERK1/2, Akt, and p38MAPK, as well as the expression of the $\alpha$-subunit of PBEF, AQP1, AQP5, and $\mathrm{ENaC}$. We found that the results of this study are in accordance with the previous literature. ${ }^{19}$ Although PBEF has been reported to inhibit the expression of $\mathrm{AQPs}$ and $\mathrm{ENaC},{ }^{8} \mathrm{PBEF}$ has a positive regulatory activity on the phosphorylation of ERK1/2, Akt, and p38MAPK, which is consistent with previous study. ${ }^{19}$ The modeling method of pulmonary edema is generally used to produce strong inflammatory reaction in pulmonary epithelial cells. Based on these studies and ours, PBEF is generally considered a proinflammatory factor, inducing inflammatory reaction leads to the reduction of 
A

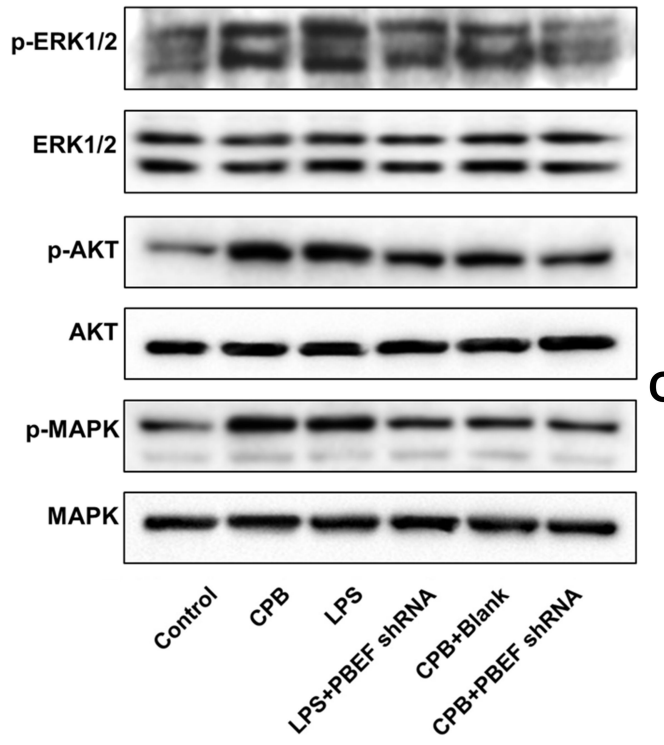

B

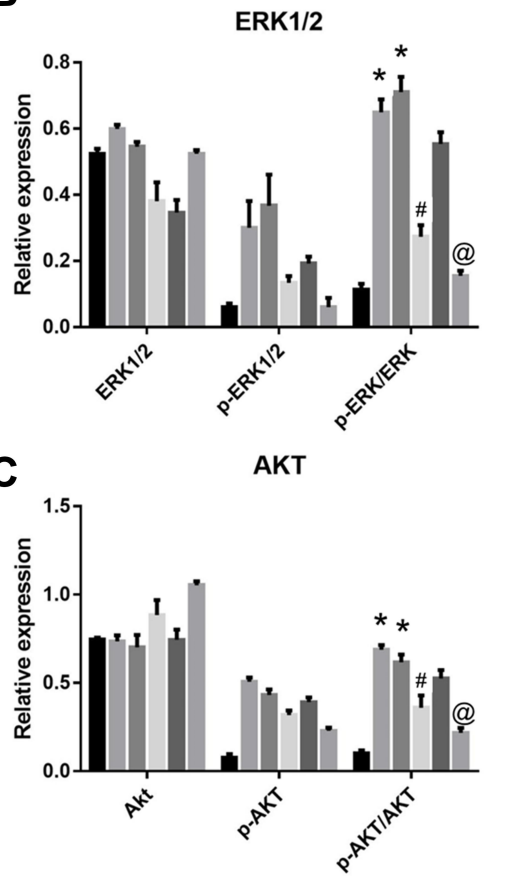

a. Control

- CPB

- LPS

- LPS+PBEF ShRNA

CPB+Blank

CPB+PBEF shRNA

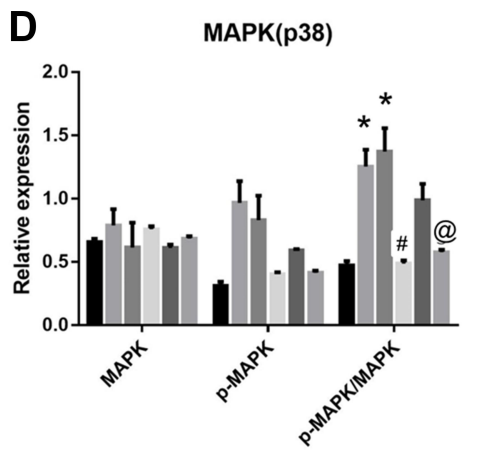

Figure 6 Adenovirus-encoding sh-PBEF reduced the increased phosphorylation of ERKI/2, Akt, and p38MAPK in rat lung tissue. (A) Representative blots; (B) Phosphorylation of ERKI/2; (C) Phosphorylation of AKT; (D) Phosphorylation of P38MAPK vs control; Original blots were shown in Supplemental figure I.*P<0.05; vs LPS, ${ }^{\#} \mathrm{P}<0.05$; vs CPB+Blank, ${ }^{@} \mathrm{P}<0.05$.

Abbreviations: PBEF, pre-B cell colony enhancing factor; $\mathrm{CPB}$, cardiopulmonary bypass.

AQPs and ENaC expression through MAPK/ERK signaling pathway. ${ }^{12,14,27}$ Postoperative pulmonary edema or serious lung injury is not inevitable, and the corresponding inflammatory response may not be very severe. We believe that the model used in this study successfully simulated the state of clinical mild lung injury.

$\mathrm{PI} 3 \mathrm{~K} / \mathrm{Akt}$, as a central signaling pathway, has multiple regulatory functions in cell survival, ${ }^{32,33}$ neuronal plasticity, ${ }^{34}$ etc. Activation of the PI3K/Akt pathway contributes to the expression of ENaC or AQPs, and reduces the symptoms of fluid accumulation in vivo. ${ }^{13,35}$ PBEF can activate both the PI3K/Akt pathway and MAPK/ERK pathway. ${ }^{8}$ In this study, under the condition of DHCA with $\mathrm{CPB}$, PBEF mainly promoted water transport in the lung through the MAPK/ERK and PI3K/Akt pathways. In a previous study, we have established a direct correlation between designated signaling pathways and alveolar epithelial sodium-water transport system using the specific inhibitors. ${ }^{8}$ This study further confirmed that MAPK/ERK and PI3K/Akt pathways might be involved in the regulation of PBEF on lung injury in rats receiving CPB.

We also detected the expression of SP in lung tissue. SP is mainly expressed in the alveoli, which helps to reduce the surface tension of the alveoli, resist inhalation of microorganisms, and reduce the function of inflammatory response on damage of alveoli. ${ }^{36}$ This study found that under CPB conditions, SP expression in the lung increased significantly, which was consistent with its protective function. Under CPB conditions, the inhibition of PBEF expression on SP expression is no longer significant. Therefore, it can be inferred that there are still other regulatory elements of $\mathrm{SP}$ and $\mathrm{ENaC}$ that are limited by the effects of PI3K/Akt and MAPK/ERK pathways.

It is worth noting that inhibition of PBEF expression led to significantly suppressed phosphorylation levels of ERK, AKT, and MAPK in both groups, and expression of AQP1, AQP5, ENaC and NKA, the sodium-water transport system protein-related factors and SP. In CPB, although the expression of aqua channel-related proteins (AQP1, AQP5) was suppressed, the expression of $\mathrm{ENaC}$ and the activity of sodium potassium pump (NKA) were slightly increased, while the expression of SP did not change. Especially, sh-PBEF down-regulated the activity caused by LPS, but not by CPB. Obviously, this point still requires future clarification. 
In this study, SP expression in lung tissue was also detected. SP is mainly expressed in the alveoli, which helps to reduce alveolar surface tension, resists inhaled microorganisms, and reduces the function of inflammation on alveolar damage. ${ }^{36}$ The expression of SP in the lungs increased significantly, corresponding to the protective function of SP. However, PEBF inhibition resulted in downregulation of SP under the condition of LPS stimulation, but not CPB. It can be inferred that there is also lung hypoxia/infiltration upstream of SP. Pressure regulation, but the regulatory elements that are limited by the ERK, AKT and MAPK pathways, are worthy of further exploration in future research.

In conclusion, PBEF could be a marker of lung injury after $\mathrm{CPB}$. Reducing PBEF expression can repair the activity of sodium-water transport system and reduce pulmonary edema after CPB, likely through reducing MAPK, ERK1/2, and Akt signaling pathways.

\section{Funding}

National Natural Science Foundation of China (81660073); Science and Technology Plan of Jiangxi Health Committee (20204337).

\section{Disclosure}

The authors report no conflicts of interest in this work.

\section{References}

1. Ziganshin BA, Elefteriades JA. Deep hypothermic circulatory arrest. Ann Cardiothoracic Surg. 2013;2(3):303-315. doi:10.3978/j. issn.2225-319X.2013.01.05

2. Amir G, Ramamoorthy C, Riemer RK, Reddy VM, Hanley FL. Neonatal brain protection and deep hypothermic circulatory arrest: pathophysiology of ischemic neuronal injury and protective strategies. Ann Thorac Surg. 2005;80(5):1955-1964. doi:10.1016/j. athoracsur.2004.12.040

3. Davidson JA, Khailova L, Treece A, et al. Alkaline Phosphatase Treatment of Acute Kidney Injury in an Infant Piglet Model of Cardiopulmonary Bypass with Deep Hypothermic Circulatory Arrest. Sci Rep. 2019;9(1):14175. doi:10.1038/s41598-019-50481-w

4. Gong M, Li L, Liu Y, et al. Moderate Hypothermic Circulatory Arrest Is Preferable During Cardiopulmonary Bypass. Ther Hypothermia Temp Manag. 2020;10(2):114-121. doi:10.1089/ther.2019.0005

5. Nagashima M, Shin'oka T, Nollert G, Shum-Tim D, Rader CM, Mayer JE. High-volume continuous hemofiltration during cardiopulmonary bypass attenuates pulmonary dysfunction in neonatal lambs after deep hypothermic circulatory arrest. Circulation. 1998;98(19 Suppl):II378.

6. Tsushima K, King LS, Aggarwal NR, De Gorordo A, D'Alessio FR, Kubo K. Acute lung injury review. Int Med. 2009;48(9):621-630. doi:10.2169/internalmedicine.48.1741

7. Butt Y, Kurdowska A, Allen TC. Acute Lung Injury: A Clinical and Molecular Review. Arch Pathol Lab Med. 2016;140(4):345-350. doi:10.5858/arpa.2015-0519-RA
8. Xu W, Zhou J, You M, et al. Pre-B-cell colony enhancing factor regulates the alveolar epithelial sodium-water transport system through the ERK and AKT pathways. Am J Transl Res. 2019;11 (9):5824-5835.

9. Wang W, Ji H-L. Epithelial Sodium and Chloride Channels and Asthma. Chin Med J. 2015;128(16):2242-2249. doi:10.4103/03666999.162494

10. Johnson MD, Widdicombe JH, Allen L, Barbry P, Dobbs LG. Alveolar epithelial type I cells contain transport proteins and transport sodium, supporting an active role for type I cells in regulation of lung liquid homeostasis. Proc Natl Acad Sci U S A. 2002;99 (4):1966-1971. doi:10.1073/pnas.042689399

11. Ma T, Fukuda N, Song Y, Matthay MA, Verkman AS. Lung fluid transport in aquaporin-5 knockout mice. J Clin Invest. 2000;105 (1):93-100. doi:10.1172/JCI8258

12. Frank J, Roux J, Kawakatsu H, et al. Transforming Growth Factor- $\beta 1$ Decreases Expression of the Epithelial Sodium Channel $\alpha \mathrm{ENaC}$ and Alveolar Epithelial Vectorial Sodium and Fluid Transport via an ERK1/2-dependent Mechanism. $J$ Biol Chem. 2003;278 (45):43939-43950. doi:10.1074/jbc.M304882200

13. Qi D, He J, Wang D, et al. 17ß-estradiol suppresses lipopolysaccharide-induced acute lung injury through PI3K/Akt/SGK1 mediated upregulation of epithelial sodium channel $(\mathrm{ENaC})$ in vivo and in vitro. Respir Res. 2014;15(1):159. doi:10.1186/s12931-014-0159-1

14. Ming G-F, Ma X-H, Xu D-M, et al. PBEF promotes the apoptosis of pulmonary microvascular endothelial cells and regulates the expression of inflammatory factors and AQP1 through the MAPK pathways. Int $J$ Mol Med. 2015;36(3):890-896. doi:10.3892/ ijmm.2015.2283

15. Luk T, Malam Z, Marshall JC. Pre-B cell colony-enhancing factor (PBEF)/visfatin: a novel mediator of innate immunity. J Leukoc Biol. 2008;83(4):804-816. doi:10.1189/jlb.0807581

16. Choi S-E, Fu T, Seok S, et al. Elevated microRNA-34a in obesity reduces NAD + levels and SIRT1 activity by directly targeting NAMPT. Aging Cell. 2013;12(6):1062-1072. doi:10.1111/acel.12135

17. Samal B, Sun Y, Stearns G, Xie C, Suggs S, McNiece I. Cloning and characterization of the cDNA encoding a novel human pre-B-cell colony-enhancing factor. Mol Cell Biol. 1994;14(2):1431-1437. doi:10.1128/MCB.14.2.1431

18. Fukuhara A, et al. Visfatin: a protein secreted by visceral fat that mimics the effects of insulin. Science. 2005;307(5708):426-430. doi:10.1126/science. 1097243

19. Deng W, Li CY, Tong J, Zhang W, Wang D-X. Regulation of ENaC-mediated alveolar fluid clearance by insulin via PI3K/Akt pathway in LPS-induced acute lung injury. Respir Res. 2012;13 (1):29. doi:10.1186/1465-9921-13-29

20. Sun Z, Lei H, Zhang Z. Pre-B cell colony enhancing factor (PBEF), a cytokine with multiple physiological functions. Cytokine Growth Factor Rev. 2013;24(5):433-442. doi:10.1016/j.cytogfr.2013.05.006

21. Ye SQ, Simon BA, Maloney JP, et al. Pre-B-Cell Colony-enhancing Factor as a Potential Novel Biomarker in Acute Lung Injury. Am $J$ Respir Crit Care Med. 2005;171(4):361-370. doi:10.1164/ recm.200404-563OC

22. Jungwirth B, Mackensen GB, Blobner M, et al. Neurologic outcome after cardiopulmonary bypass with deep hypothermic circulatory arrest in rats: description of a new model. $J$ Thorac Cardiovasc Surg. 2006;131(4):805-812. doi:10.1016/j.jtcvs.2005.11.017

23. Liu G, Lv H, An Y, Wei X, Yi X, Yi H. Tracking of transplanted human umbilical cord-derived mesenchymal stem cells labeled with fluorescent probe in a mouse model of acute lung injury. Int $\mathrm{J} \mathrm{Mol}$ Med. 2018;41(5):2527-2534. doi:10.3892/ijmm.2018.3491

24. Kumari N, Thakur N, Cho HR, Choi SH. Assessment of Early Therapeutic Response to Nitroxoline in Temozolomide-Resistant Glioblastoma by Amide Proton Transfer Imaging: A Preliminary Comparative Study with Diffusion-weighted Imaging. Sci Rep. 2019;9(1):5585. doi:10.1038/s41598-019-42088-y 
25. Yang W, Zeng Y, Li B, et al. Pre-B-cell colony enhancing factor (PBEF) increases endothelial permeability in hypoxia/re-oxygenation model. Int J Clin Exp Med. 2015;8(6):8842-8847.

26. Yan N, Yang W, Dong X, et al. Promotion of anoxia-reoxygenationinduced inflammation and permeability enhancement by nicotinamide phosphoribosyltransferase-activated MAPK signaling in human umbilical vein endothelial cells. Exp Ther Med. 2017;14 (5):4595-4601. doi:10.3892/etm.2017.5083

27. Fang Q, You M, Xu W, Yang W, Gong Y, Dong X. pre-B cell colony enhancing factor negatively regulates $\mathrm{Na}+$ and fluid transport in lung epithelial cells. Am J Transl Res. 2018;10(7):2047-2054.

28. Waterbury T, Clark TJ, Niles S, Farivar RS. Rat model of cardiopulmonary bypass for deep hypothermic circulatory arrest. $J$ Thorac Cardiovasc Surg. 2011;141(6):1549-1551. doi:10.1016/j. jtcvs.2011.01.062

29. Song Z, Shen F, Zhang Z, Wu S, Zhu G. Calpain inhibition ameliorates depression-like behaviors by reducing inflammation and promoting synaptic protein expression in the hippocampus. Neuropharmacology. 2020;174:108175. doi:10.1016/j. neuropharm.2020.108175

30. Lei J, Wei Y, Song P, et al. Cordycepin inhibits LPS-induced acute lung injury by inhibiting inflammation and oxidative stress. Eur J Pharmacol. 2018;818:110-114. doi:10.1016/j.ejphar.2017.10.029

31. Berglund L, Bjorling E, Oksvold P, et al. A genecentric Human Protein Atlas for expression profiles based on antibodies. Mol Cell Proteomics. 2008;7(10):2019-2027. doi:10.1074/mcp.R800013MCP200
32. Zhu G, Liu Y, Wang Y, Bi X, Baudry M. Different patterns of electrical activity lead to long-term potentiation by activating different intracellular pathways. $J$ Neurosci. 2015;35(2):621-633. doi:10.1523/JNEUROSCI.2193-14.2015

33. Zhu G, Wang X, Wu S, Li X, Li Q. Neuroprotective Effects of Puerarin on 1-methyl-4-phenyl-1,2,3,6-tetrahydropyridine induced Parkinson's Disease Model in Mice. Phytother Res. 2014;28 (2):179-186. doi:10.1002/ptr.4975

34. Song Z, Chen $\mathrm{H}, \mathrm{Xu} \mathrm{W}, \mathrm{Wu} \mathrm{S}, \mathrm{Zhu} \mathrm{G}$. Basolateral amygdala calpain is required for extinction of contextual fear-memory. Neurobiol Learn Mem. 2018;155:180-188. doi:10.1016/j.nlm.2018.08.004

35. Song D, Liu X, Diao Y, et al. Hydrogen-rich solution against myocardial injury and aquaporin expression via the PI3K/Akt signaling pathway during cardiopulmonary bypass in rats. Mol Med Rep. 2018;18(2):1925-1938. doi:10.3892/mmr.2018.9198

36. Nathan N, Taytard J, Duquesnoy P, et al. Surfactant protein A: A key player in lung homeostasis. Int J Biochem Cell Biol. 2016;81(Pt A):151-155. doi:10.1016/j.biocel.2016.11.003

\section{Publish your work in this journal}

Drug Design, Development and Therapy is an international, peerreviewed open-access journal that spans the spectrum of drug design and development through to clinical applications. Clinical outcomes, patient safety, and programs for the development and effective, safe, and sustained use of medicines are a feature of the journal, which has also been accepted for indexing on PubMed Central. The manuscript management system is completely online and includes a very quick and fair peer-review system, which is all easy to use. Visit http://www. dovepress.com/testimonials.php to read real quotes from published authors. 